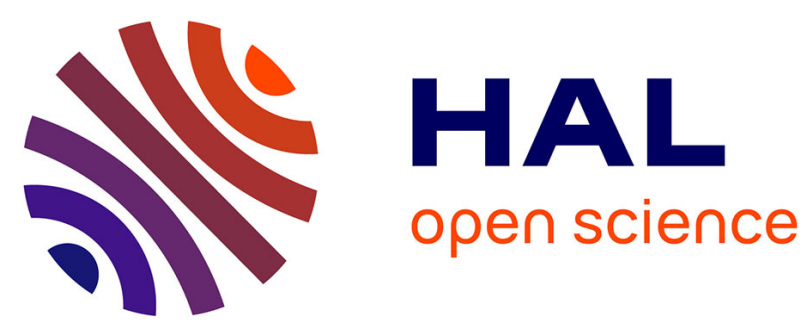

\title{
Deterioration modelling of contact surfaces for a friction drive system
}

\author{
Diego Jair Rodriguez Obando, John Jairo Martinez Molina, Christophe \\ Bérenguer
}

\section{- To cite this version:}

Diego Jair Rodriguez Obando, John Jairo Martinez Molina, Christophe Bérenguer. Deterioration modelling of contact surfaces for a friction drive system. ESREL 2016 - 26th European Safety and Reliability Conference, University of Strathclyde, UK Safety and Reliability Society and European Safety and Reliability Association-ESRA, Sep 2016, Glasgow, United Kingdom. pp.1961-1968. hal01376794

\section{HAL Id: hal-01376794 \\ https://hal.science/hal-01376794}

Submitted on 6 Oct 2016

HAL is a multi-disciplinary open access archive for the deposit and dissemination of scientific research documents, whether they are published or not. The documents may come from teaching and research institutions in France or abroad, or from public or private research centers.
L'archive ouverte pluridisciplinaire HAL, est destinée au dépôt et à la diffusion de documents scientifiques de niveau recherche, publiés ou non, émanant des établissements d'enseignement et de recherche français ou étrangers, des laboratoires publics ou privés. 


\title{
Deterioration modelling of contact surfaces for a friction drive system
}

\author{
D.J. Rodriguez \& J.J. Martinez \& C. Berenguer \\ Univ. Grenoble Alpes, GIPSA-lab, F-38000 Grenoble, France \\ CNRS, GIPSA-lab, F-38000 Grenoble, France
}

\begin{abstract}
This article presents a novel model of surface deterioration for a friction drive. For this basic transmission system, the difference between tangential speeds is linked with the production of contact force and at the same time with an energy transfer that deteriorates the contact surfaces. Thus, an optimal management between health state of components and use of energy resources is required. In a first step, the physics of the system is modeled in a deterministic way. Then, considering either random system parameters or random system inputs and usage, a complete stochastic model for the system deterioration is proposed. The developed model allows to link the deterioration evolution to the inputs and operating modes of the system; to assess the behavior of deterioration in critical stages, such as sharp motion and smooth motion. The advantages to design and to analyze such a model are finally discussed in terms of reliability-adaptive systems.
\end{abstract}

\section{INTRODUCTION}

The Remaining Useful Life (RUL) of an asset or system is defined as the time left from the current time to the end of the useful life. Prognosis of RUL is a widely studied topic in health management of resources for automated engineered. RUL prognosis has also an important role in the management of energy resources for e.g. autonomous devices. A good overview over existing methods for RUL prognosis is given in ( $\mathrm{Si}$, Wang, Hu, \& Zhou 2011). Note that the meaning of "useful life" varies according to the studied engineering field, thus the owner of the asset is the one who defines the sense of useful life and its management.

Deterioration of components and failures affects directly the lifetime of assets, and consequently their usability and/or productivity. On the other hand, energy consumption is another critical variable to manage in relation with the deterioration behavior. For this reason, nowadays manufacturers or end users are becoming increasingly motivated to manage the complete life-cycle of an asset and to optimize the production process and its energy consumption using proactive strategies. Thus, an optimal management between health state of components and use of energy resources is required, which is defined in this paper as a "RUL-aware" operation.

The aim of this work is to develop a mathematical model for the deterioration of the contact surfaces of a friction drive system, which is a type of transmission that uses two circular devices to transfer mechanical power by friction, used e.g. in some electrical bikes with two main components: wheel and DC-motor. The basic model of friction drive transmission relies on the assumption of equal tangential speeds of wheel and motor surfaces. However, the difference between speeds can not be always neglected because this is linked with the efficiency of mechanical transmission that drives the wheel and the production of contact force, which is associated with a transfer of energy that also deteriorates the contact surfaces. Then, the energy transfer is at the same time, the useful phenomenon and the source of deterioration and as such it requires to be properly modeled; thus, allowing an optimal control ensures both a sufficient drive power and an acceptable deterioration. As explained in Section 4, this work can then be seen as a required step towards developing a complete Reliability Adaptive Systems or RAS according to (Meyer \& Sextro 2014).

We consider a special case of a friction drive system (roller-on-tire system) and we develop an analytical physics-based model for the deterioration of the driving contact surfaces. The developed model integrates the deterioration as one of the states of the system, thus it allows: a) to link the deterioration evolution to the inputs and operating modes of the system, b) to assess the behavior of deterioration in critical stages, such as sharp motion and smooth motion and c) to predict analytically an interval of useful lifetime before the total-failure-time of actuator, taking into account stochastic perturbations. 


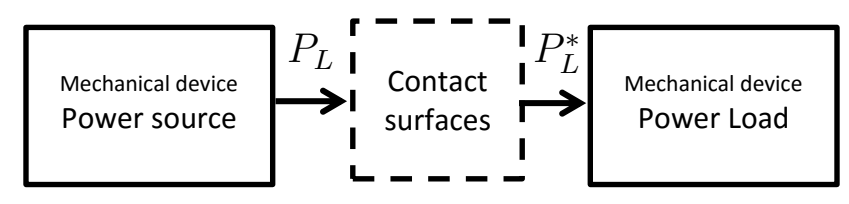

Figure 1: Losses of power in contact

The remainder of this paper is organized as follows. Section 2 presents the design and analysis of the deterioration model of the considered friction drive system. Section 2.1 contains the general definition of the particular friction drive which here is called "roller-on-tire" system. In a first step, in Section 2.2, the physics of the system, and in particular the link between deterioration dynamics and energy transfer at the friction surface, is modeled in a deterministic way. In Section 2.3 an useful space state representation is presented. Then, considering either random system parameters or random system inputs and usage, a complete stochastic model for the system deterioration is proposed in Section 2.4.

Section 3 shows the analysis of simulations for a case study. Finally, plans for future work directions are given in Section 4 in the framework of RAS.

\section{FRICTION DRIVE SYSTEM MODELLING}

Figure 1 presents the basic principles of the mechanical devices considered in this work: a power transmitted from the source to the load, $P_{L}$, is higher than the power which actually causes the motion, $P_{L}^{*}$. This phenomenon is modeled under the general hypothesis that the loss of efficiency in the conversion from energy to motion can be an estimable image of the deterioration of materials in contact.

\subsection{Roller-on-tire system description}

The "roller-on-tire" system is a friction drive composed by a driven device and a motor, as depicted in figure 2, for which it is supposed that: a) both contact surfaces, the rotor of the motor and the external part of the driven device, deteriorate, b) both surface deterioration of the motor and the tire reach eventually a threshold above which the system is considered failed, and c) an inversion of motion sense cannot occur at any time.

Symbols of the equations and units are shown in Table 1.

\subsection{Analytical physics-based modelling}

The mechanical analysis is based on the Dahl model and LuGre model of friction, which are widely studied in the literature of mechanics. A good overview about those models can be read in (Geffen 2009) and (Armstrong-Helouvry \& Canudas de Wit 1996). These models represent the friction force with the components of the Stribeck friction effect, for which

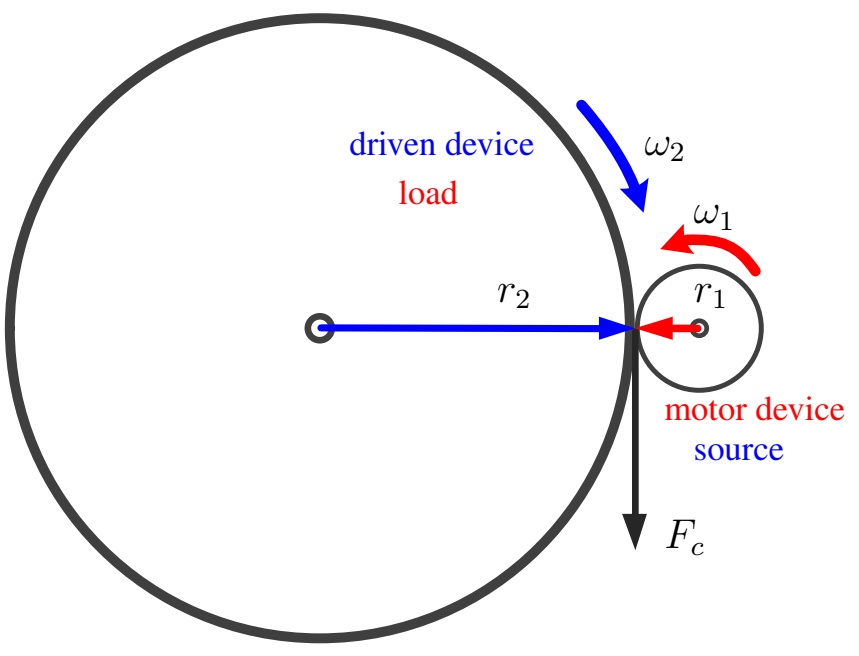

Figure 2: Basic roller-on-tire motor system

Table 1: Parameters, units and symbols

\begin{tabular}{|c|c|c|}
\hline Symbol & Units & Physical meaning \\
\hline$v_{1}$ & $m / s$ & Tangential speed of the motor \\
\hline$v_{2}$ & {$[\mathrm{~m} / \mathrm{s}]$} & Tangential speed of the driven device \\
\hline$\omega_{1}$ & {$[\mathrm{rad} / \mathrm{s}]$} & Angular speed of the motor \\
\hline$\omega_{2}$ & {$[\mathrm{rad} / \mathrm{s}]$} & Angular speed of the driven device \\
\hline$\dot{\omega}_{1}$ & {$\left[\mathrm{rad} / \mathrm{s}^{2}\right]$} & Angular acceleration of the motor \\
\hline$\dot{\omega_{2}}$ & {$\left[\mathrm{rad} / \mathrm{s}^{2}\right]$} & Angular acceleration of the driven device \\
\hline$r_{1}$ & {$[m]$} & External radius of the motor \\
\hline$r_{2}$ & {$[m]$} & External radius of the driven device \\
\hline$B_{1}$ & {$\left[\mathrm{Kgm}^{2} / \mathrm{s}\right]$} & Viscous damping coefficient of the motor \\
\hline$B_{2}$ & {$\left[\mathrm{Kgm}^{2} / \mathrm{s}\right]$} & Viscous damping coefficient of the driven device \\
\hline$J_{1}$ & {$\left[\mathrm{Kgm}^{2}\right]$} & Moment of inertia of the motor \\
\hline$J_{2}$ & {$\left[\mathrm{Kgm}^{2}\right]$} & Moment of inertia of the driven device \\
\hline$T_{m}$ & {$[\mathrm{Nm}]$} & Torque of the motor \\
\hline$T_{L}$ & {$[\mathrm{Nm}]$} & Load torque seen by the motor \\
\hline$T_{L}^{*}$ & {$[\mathrm{Nm}]$} & Source torque observed by the driven device \\
\hline$K_{m}$ & {$[\mathrm{Vs} / \mathrm{rad}]$} & Motor back electromotive force constant \\
\hline$I$ & & Electrical current of the motor \\
\hline$\alpha$ & {$[\mathrm{Ns} / \mathrm{m}]$} & Contact quality coefficient \\
\hline
\end{tabular}

the friction force has an inverse relation with respect to the speed; the Coulomb friction, for which the force remains as a constant with respect to the speed at the beginning of motion; and the viscous friction, for which the friction force is proportional to the speed. In the present contribution, Stribeck friction effect and Coulomb friction are considered negligible; that is, only the viscous friction is taken into account, due to the fact that it does not exist any possibility of inversion of the motion sense.

Here $F_{c}$ is assumed as a frictional force, tangential to both the motor and the driven devices, which causes a rotational torque over the driven device. A similar approximation of the law of friction by a continuous function of velocity is analysed in (Popov 2010). Consequently, the friction force component depicted in Equation (1), depends on the difference $\Delta_{v}$ between the tangential speeds of motor and driven device, $v_{1}$ and $v_{2}$ respectively, and the coefficient $\alpha$, which in this paper is called contact quality coefficient and that corresponds to the viscous friction coefficient in the classic context of friction components modelling.

$F_{c}=\alpha\left(v_{1}-v_{2}\right)=\alpha\left(r_{1} \omega_{1}-r_{2} \omega_{2}\right)=\alpha \Delta_{v}$

The parameter $\alpha$ is classically considered to be constant, but in this paper it is treated as a timevarying parameter. In some sense, this $\alpha$ gives an initial idea about the quality of contact between both 
rolling devices in each time of the motion. Then, because of the frictional phenomena, the condition of contact depends on the deterioration of materials and varies with time, i.e. $\alpha=f(D, t) \succ 0$.

Thus both the torque produced by the motor and transferred to the load $T_{L}(t)$ and the torque observed by the driven device $T_{L}^{*}(t)$ depend on $F_{c}(t)$ according to equations (2) and (3). In this paper, dependence on time is not written for convenience.

$T_{L}=F_{c} r_{1}$

$T_{L}^{*}=F_{c} r_{2}$

The system dynamics using Newton's laws of motion can be written as follows:

$$
\begin{aligned}
& \Sigma T_{1}=J_{1} \dot{\omega}_{1}=T_{m}-T_{L}-B_{1} \omega_{1} \\
& \dot{\omega}_{1}=\frac{1}{J_{1}}\left(K_{m} I-r_{1} \alpha\left(r_{1} \omega_{1}-r_{2} \omega_{2}\right)-B_{1} \omega_{1}\right) \\
& \Sigma T_{2}=J_{2} \dot{\omega}_{2}=T_{L}^{*}-B_{2} \omega_{2} \\
& \dot{\omega}_{2}=\frac{1}{J_{2}}\left(r_{2} \alpha\left(r_{1} \omega_{1}-r_{2} \omega_{2}\right)-B_{2} \omega_{2}\right)
\end{aligned}
$$

Equations (4) and (5) can be rewritten in terms of power multiplying them by $\omega_{1}$ and $\omega_{2}$ respectively as follows:

$$
\begin{aligned}
& J_{1} \dot{\omega}_{1} \omega_{1}=K_{m} I \omega_{1}-T_{L} \omega_{1}-B_{1} \omega_{1}^{2} \\
& J_{2} \dot{\omega}_{2} \omega_{2}=T_{L}^{*} \omega_{2}-B_{2} \omega_{2}^{2}
\end{aligned}
$$

where terms in equations are shown in Table 2:

Table 2: Terms of power equations

\begin{tabular}{lll}
\hline Term & Physical meaning & Symbol \\
\hline$J_{1} \dot{\omega}_{1} \omega_{1}$ & Total power in the motor & $P_{T M}$ \\
$K_{m} I \omega_{1}$ & Electric power in the motor & $P_{\text {motor }}$ \\
$T_{L} \omega_{1}$ & Power transferred to the load & $P_{L}$ \\
$B_{1} \omega_{1}^{2}$ & Power caused by viscous damping $B_{1}$ & $P_{B 1}$ \\
$J_{2} \omega_{2} \omega_{2}$ & Total power for the driven device & $P_{T W}$ \\
$T_{L}^{*} \omega_{2}$ & Power caused by contact & $P_{L}^{*}$ \\
& observed by the driven device & \\
$B_{2} \omega_{2}^{2}$ & Power caused by viscous damping $B_{2}$ & $P_{B 2}$ \\
\hline
\end{tabular}

Taking into account Equations (6) and (7), power in contact $P_{c}$ can be expressed as follows:

$$
\begin{aligned}
& P_{c}=P_{L}-P_{L}^{*}=T_{L} \omega_{1}-T_{L}^{*} \omega_{2} \\
& P_{c}=F_{c}\left(r_{1} \omega_{1}-r_{2} \omega_{2}\right)=F_{c} \Delta_{v}
\end{aligned}
$$

Using Equation (1) this takes the form:

$P_{c}=\alpha\left(r_{1} \omega_{1}-r_{2} \omega_{2}\right)^{2}=\alpha \Delta_{v}^{2}$

We define the dynamic efficiency of the transmission $\eta_{c}(t)$ as:

$\eta_{c}=P_{L}^{*} / P_{L}=\left(P_{L}-P_{c}\right) / P_{L}$ which is equivalent to:

$\eta_{c}=F_{c} r_{2} \omega_{2} / F_{c} r_{1} \omega_{1}=\frac{r_{2}}{r_{1}} \frac{\omega_{2}}{\omega_{1}}=\frac{v_{2}}{v_{1}}$

We assume that it is possible to measure the speeds $v_{1}$ and $v_{2}$ (or equivalently, $\omega_{1}$ and $\omega_{2}$ ) in this kind of mechanisms. Thus $\eta$ can be computed dynamically.

The energy dissipated in the contact $E_{c}$ between the surfaces in roller-on-tire system can be obtained from (9). Thus $E_{c}$ depends on the speed between the surfaces as follows:

$E_{c}=\int_{t_{1}}^{t_{2}} P_{c} d t=\int_{t_{1}}^{t_{2}} \alpha \Delta_{v}^{2} d t$

The dissipated energy could be considered as an image of the heat and material losses produced at the contact level during traction, as considered Similar conclusion were found by (Meyer \& Sextro 2014). Thus, if heat losses are neglected, deterioration of surfaces $D_{c}$ can be considered as proportional to the dissipated energy in the contact:

$D=c E_{c}=c \int_{t_{1}}^{t_{2}} P_{c} d t=c \int_{t_{1}}^{t_{2}} \alpha \Delta_{v}^{2} d t$

where $c$ is a proportionality factor that relates deteriorated material because of friction with the energy dissipated. That is, here the deterioration is considered as an image of the dissipated energy at the contact level during power transmission.

Equation (13) shows that the dynamics of the system and the contact surfaces deterioration are linked through $\alpha$. From Equation (13) and since $\alpha=$ $f(D)$, a nonlinear variation of the deterioration with respect to time according can be obtained:

$\dot{D}=c \alpha \Delta_{v}^{2}=H_{\text {nonlinear }}\left(\omega_{1}, \omega_{2}, D\right)$

At the same time, $\alpha$ varies depending on the deterioration of materials. It is assumed that the bigger $D$, the lower $\alpha$ and that $D$ is monotonically increasing and bounded until $D_{\max }$. We define the maximum value of deterioration $D_{\max }$, as the value of $D$ in which the system is considered completely failed. In this paper, it is considered that the evolution of $\alpha$ depends on the evolution of the deterioration $D$ as depicted in Figure 3. Consequently, a first order linear variation of $\alpha$, with respect to $D$ with slope $m>0$, $m \in \mathbb{R}^{+}$and initial value $b>0, b \in \mathbb{R}^{+}$, is defined as:

$\alpha=-m D+b$

From Equations (14) and (15), we get:

$$
\begin{aligned}
& \dot{D}=(-c m D+c b)\left(r_{1} \omega_{1}-r_{2} \omega_{2}\right)^{2} \\
& \dot{D}=-m \underbrace{c \Delta_{v}^{2}}_{d(t)} D+b \underbrace{c \Delta_{v}^{2}}_{d(t)}
\end{aligned}
$$




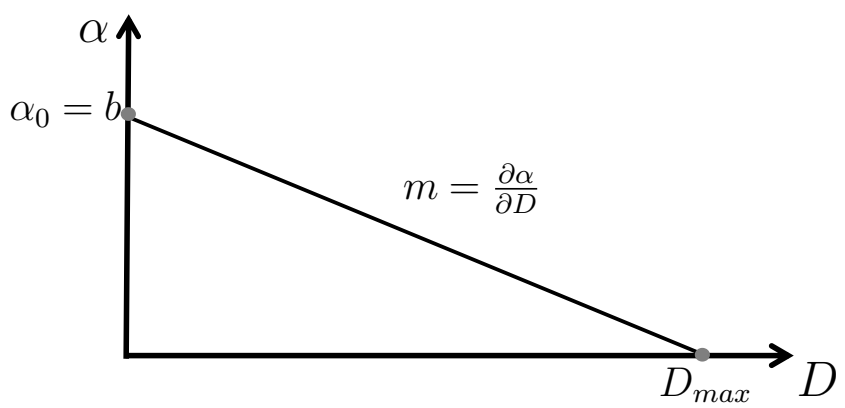

Figure 3: $\alpha$ as a function of $D$

Taking into account the term $d(t)=c\left(r_{1} \omega_{1}-\right.$ $\left.r_{2} \omega_{2}\right)^{2}$, Equation (17) can be rewritten as follows:

$\dot{D}=-m d(t) D+b d(t)$

We can compute the maximum value of the deterioration with respect to time $D_{\max }$ as:

$D_{\max } \triangleq \lim _{t \rightarrow+\infty} D(t)$

This can be calculated using Equation (18) with $\dot{D}=$ 0 , thus:

$-m D_{\max }+b=0$

$D_{\text {max }}=b / m=\alpha(0) / m$

In a complementary way, we define the normalized deterioration as:

$\bar{D} \triangleq D(t) / D_{\max }=(m / \alpha(0)) D(t)$

From Equation (15), we get the special form:

$D(\alpha)=(b-\alpha) / m=(\alpha(0)-\alpha) / m$

Since $\Delta_{v}=v_{1}-v_{2}=r_{1} \omega_{1}-r_{2} \omega_{2}$ and using (4) and (5):

$$
\begin{aligned}
\dot{\Delta_{v}}=\left(r_{1} / J_{1}\right)\left(K_{m} I\right. & \left.-\alpha r_{1} \Delta_{v}-B_{1} \omega_{1}\right) \\
& -\left(r_{2} / J_{2}\right)\left(\alpha r_{2} \Delta_{v}-B_{2} \omega_{2}\right)
\end{aligned}
$$

Equation (24) shows that it is possible to estimate the value of $\alpha$ from the measured speeds (recall that measuring the speeds is considered feasible in this kind of mechanisms), and assuming the other parameters of the systems as constant. Let denote this estimated value as $\hat{\alpha}$. Therefore using Equation (23), the deterioration $\hat{D}$ can be estimated by:

$\hat{D}=(\alpha(0)-\hat{\alpha}) / m$

Consequently, using equations (21), (22) and (25) it is obtained the normalized estimation of deterioration $\hat{\bar{D}}$ :

$\hat{\bar{D}}=\hat{D} / D_{\max }=(\alpha(0)-\hat{\alpha}) / \alpha(0)$
Deriving Equation (23) with respect to time, and using Equation (14), we get:

$\dot{D}=-(1 / m) \dot{\alpha}$

$\dot{\alpha}=-m c \dot{D}=-m c \alpha \Delta_{v}^{2}$

Equation (28) can be rewritten taking into account Equation (9):

$\alpha=-m c \int_{t_{1}}^{t_{2}} P_{c} d t$

Therefore:

$\alpha=-m c E_{c}+\alpha(0)$

Summarizing, assuming a monotonically increasing of deterioration, the proposed model shows that roller-on-tire system reaches a maximum value of deterioration $D_{\max }$, above which the transmission is consider failed, which depends on the initial value of the contact quality coefficient $\alpha(0)$ and the slope $m$, which represents the variation of $\alpha(0)$ with respect to deterioration of material $D$. At the same time the normalized estimation of deterioration $\hat{\bar{D}}$ can be obtained from $\alpha(0)$ and its estimation $\hat{\alpha}$. The estimated coefficient $\hat{\alpha}$ can measure the loss of material or the modification of the surface properties at the contact level, i.e. the material that allows the contact (or the existence of mechanical transmission). At the same time $\hat{\alpha}$ establishes a relation between material of contact and energy of contact.

\subsection{State-space model for the roller-on-tire system}

In accordance with the above, roller-on-tire system is an uncertain system which depends on time-varying unknown-but-bounded uncertain parameters. Taking into account the states:

$x=\left(\begin{array}{c}\omega_{1} \\ \omega_{2} \\ D\end{array}\right)$,

the control input $u=I$ and the output $y$, the representation of the system can be expressed as:

$\left\{\begin{array}{l}\dot{x}=f(x)+B u \\ y=C x\end{array}\right.$

$B$ is an input matrix and $C$ the output matrix. In an explicit form, the representation of the system is:

$f(x)=\left(\begin{array}{c}-\frac{1}{J_{1}}\left(B_{1}+\alpha r_{1}^{2}\right) \omega_{1}+\frac{1}{J_{1}} \alpha r_{1} r_{2} \omega_{2} \\ \frac{1}{J_{2}} \alpha r_{2} r_{1} \omega_{1}-\frac{1}{J_{2}}\left(B_{2}+\alpha r_{2}^{2}\right) \omega_{2} \\ c \alpha\left(r_{1} \omega_{1}-r_{2} \omega_{2}\right)^{2}\end{array}\right)$

$B=\left[\begin{array}{c}\frac{K_{m}}{J_{1}} \\ 0 \\ 0\end{array}\right] ; C=\left[\begin{array}{ccc}1 & 0 & 0 \\ 0 & 1 & 0 \\ 0 & 0 & 1\end{array}\right] ; u=I$ 
Remark that in this case the non-linearity of the system is due to the introduction of the state $\mathrm{D}$.

\subsection{Introducing randomness in the model}

Randomness in the model can be introduced in two complementary modes: a) internal, to model the uncertainty on the parameters $m$ and $b$ (which are used to relate $a$ with $D$ ) for a given system, and the systemto-system variability and b) external, to model the variability of the operating conditions and the usage system, in this case taking into account the input $u$.

\subsubsection{Uncertain/random parameters}

In Equation (15) $b \geq 0$ and $m \geq 0$ are considered as unknown parameters, but which belong to a given known interval taking into account real applications. Thus a normal distribution is introduced to represent them:

$$
\begin{aligned}
& m \sim \mathcal{N}\left(m_{m}, \sigma_{m}^{2}\right), m_{m}>0 \\
& b \sim \mathcal{N}\left(b_{m}, \sigma_{b}^{2}\right), b_{m}>0
\end{aligned}
$$

where $m_{m}$ and $\sigma_{m}$ are the mean value and the standard deviation of the slope $m$ respectively, and $b_{m}$ and $\sigma_{b}$ are the mean value and the standard deviation of $b$ respectively.

As depicted in the shaded area in Figure 4 and according to Equations (15) and (35), the behavior of $\alpha$ with respect to $D$ constitutes a bi-dimensional space of variation. The solid line inside the shaded area is an example of probable behavior.

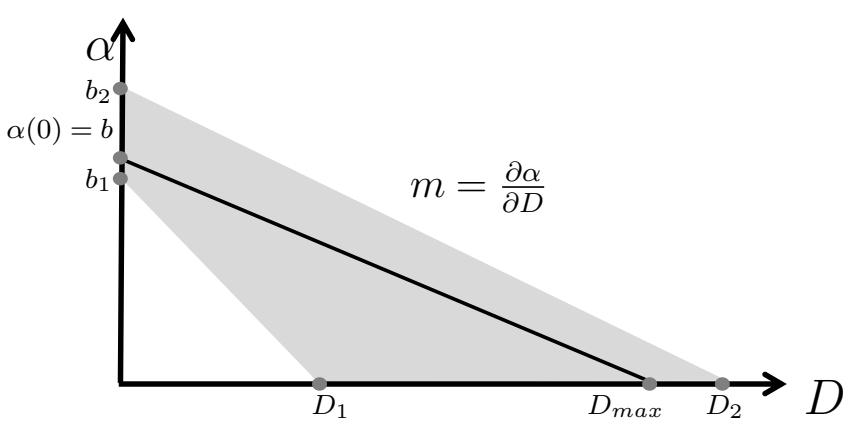

Figure 4: $\alpha$ as a function of $D$

\subsubsection{Variable operating conditions and usage}

The developed model is single input $u=I$. In this case, the variability of the operating conditions and the usage system is represented as the variation of $I$.

Consider an input $I$ as a square signal. The times $t_{h}$, in which $I$ is in high and $t_{l}$, in which $I$ is in low can vary randomly. Thus an exponential distribution is introduced to represent it:

$$
\begin{aligned}
& t_{l} \sim \operatorname{Exp}\left(1 / \mu_{t l}\right), 0<\mu_{t l} \\
& t_{h} \sim \operatorname{Exp}\left(1 / \mu_{t h}\right), 0<\mu_{t l}<\mu_{t h}
\end{aligned}
$$

where $\mu_{t l}$ and $\mu_{t h}$ are the mean values of $t_{l}$ and $t_{h}$ respectively.

The deterministic operational mode, the internal mode of randomness and the external mode of randomness are used in a case study analysis in Section 3 .

\section{CASE STUDY ANALYSIS}

To choose the parameters for simulations in order to validate the usefulness of the model, the contributions of (Huang \& Nagurka 2003) and (Rodriguez 2014) were analyzed. In the first article, a similar workbench has a built-in bike wheel to model viscous and dry friction at the wheel bearing. The second contribution is a master-thesis developed in GIPSA-Lab (CNRSFrance, Grenoble-INP, University Grenoble Alpes), which analyses the implementation of a real friction drive in an electrical bicycle. Table 3 shows the chosen parameters.

\begin{tabular}{lll}
\multicolumn{3}{l}{ Table 3: Chosen parameters } \\
\hline Parameter & Value & Unit \\
\hline$B_{1}$ & $6.36 \times 10^{-3}$ & {$\left[\mathrm{Kgm}^{2} / \mathrm{s}\right]$} \\
$B_{2}$ & $1.76 \times 10^{-3}$ & {$\left[\mathrm{Kgm}^{2} / \mathrm{s}\right]$} \\
$J_{1}$ & $3.47 \times 10^{-4}$ & {$\left[\mathrm{Kgm}^{2}\right]$} \\
$J_{2}$ & 0.2 & {$\left[\mathrm{Kgm}^{2}\right]$} \\
$r_{1}$ & 0.0315 & $\mathrm{~m}$ \\
$r_{2}$ & 0.35 & $\mathrm{~m}$ \\
$K_{m}$ & 0.0477465 & {$[\mathrm{Vs} / \mathrm{rad}]$} \\
\hline
\end{tabular}

\subsection{Deterministic operational mode}

\subsubsection{Sharp stage of motion}

The system model is simulated with an input step signal $I$ with amplitude $=20 \mathrm{~A}$. Figure 5 shows the behavior of the power in the system.

Here, $P_{L}$ the transferred power to the load, and $P_{L}^{*}$, the power seen by the driven device have a high value only at the beginning, and such as it was supposed $P_{L}>P_{L}^{*}$. The difference between $P_{L}$ and $P_{L}^{*}$ reflects the loss in contact, primarily at the beginning of motion, i.e. the sharp stage.

Remark that $P_{L}$ and $P_{L}^{*}$ reach a peak in the sharp stage, and after they drop; while $P_{\text {motor }}$, the electrical power of the motor, and $P_{B 1}$, the power that appears as a result of the viscous damping parameter $B_{1}$ reach high and near values. $P_{L}^{*}$ and $P_{B 2}$ are much lower because they depend on $\omega_{2}$ which is lower than $\omega_{1}$. Here, it is possible to see that there is a considerable influence of the viscous damping parameter $B_{1}$ in the consumption of energy, i.e. the motor must provide more power to drive its own viscous damping parameter $B_{1}$.

Figure 6 shows the behavior of the difference of power for given values of $\alpha$ with respect to time. Experimentally, it was found a maximum peak of this difference, i.e. the minimum efficiency $\eta$ when $\alpha$ is 
equal to 5.25. It means that the increasing of $\alpha$ tends to give lower losses but it do not necessarily produces the highest peak of losses. These dynamic changes reflect the importance to estimate and possibly to control the value of $\alpha$.

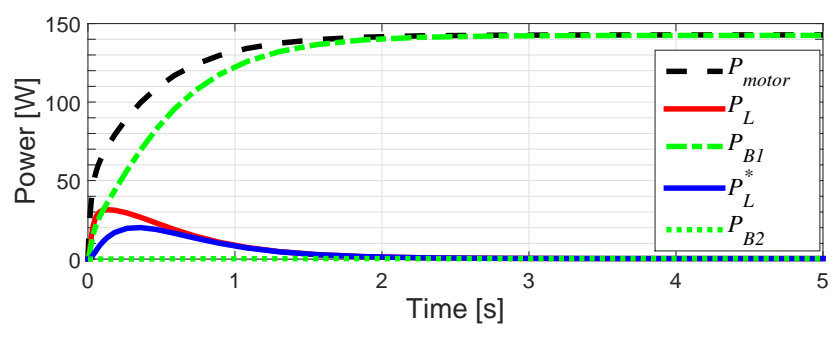

Figure 5: Behavior of power in roller-on-tire system

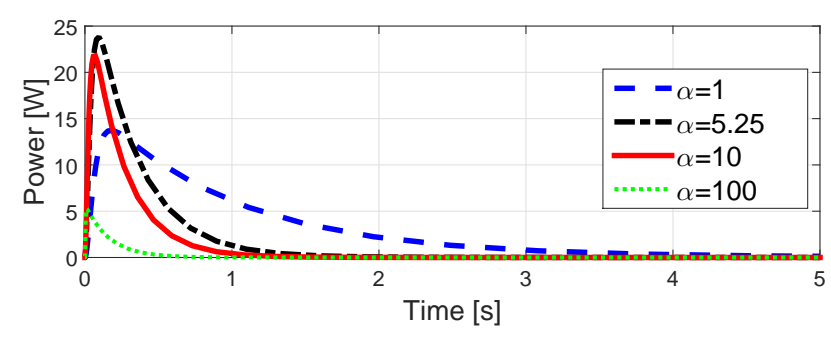

Figure 6: Loss between $P_{L}$ and $P_{L}^{*}$ for given values of $\alpha$

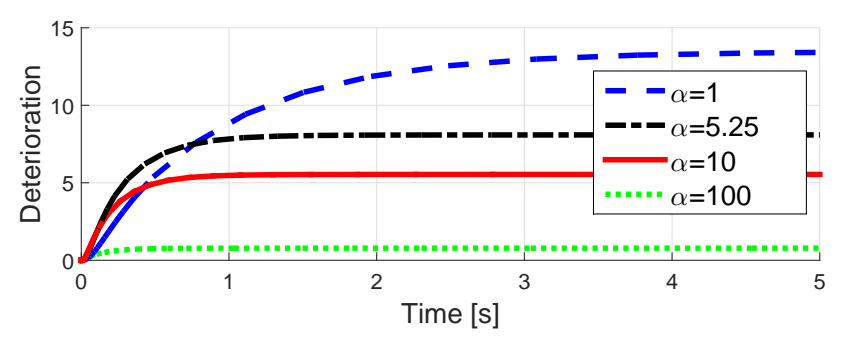

Figure 7: Deterioration for given values of $\alpha$ for sharp stage of motion. The higher the $\Delta_{v}$, the higher the deterioration of material.

Figure 7 shows the behavior of the instantaneous $D$ for a given parameter $c=1$ for the same given values of $\alpha$ than the figure 6. It is evident that $D$ is always increasing and that the highest values are related with power losses. It is also remarkable that the highest consumption of contact energy (material of contact) happen in the first seconds for every value of $\alpha$, i.e. the sharp stage of motion when also $\Delta_{v}$ is higher.

\subsubsection{Smooth stage of motion}

Given values $c=1, m=0.01$ and $b=10$ are determined arbitrarily in the framework of possible implementation and taking into account previous simulations in order to introduce the variation of $\alpha$ according to Equation (15). Figure 8 shows the behavior of states $v_{1}, v_{2}$ and $D$. Here is possible to see the behavior of deterioration at the smooth stage of motion, i.e. when $\Delta_{v}$ is lower, until the total failure. Deterioration reaches the $D_{\max }$ value at 300 hours of use for the given scenario.

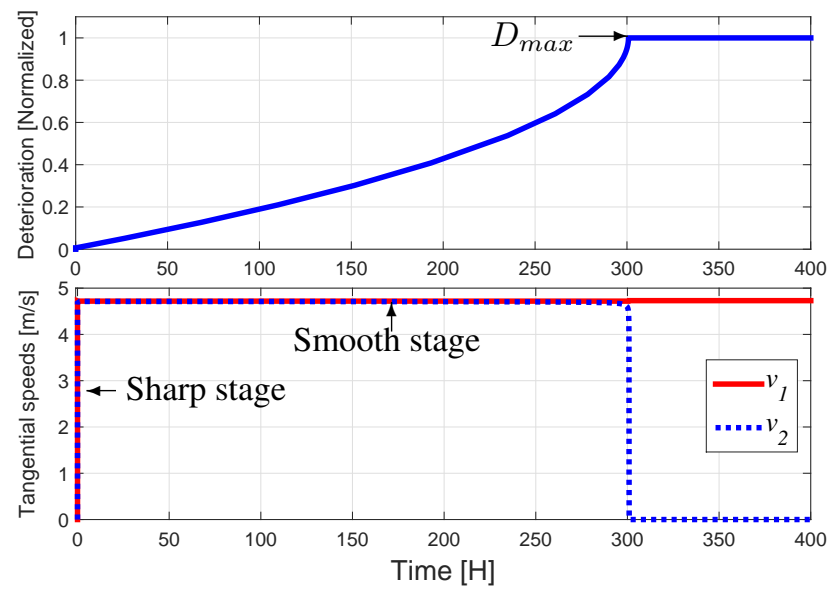

Figure 8: Deterioration $D$, tangential speed of motor $v_{1}$ and tangential speed of wheel $v_{2}$ for a step input. It shows the failure of system at $300 \mathrm{~h}$.
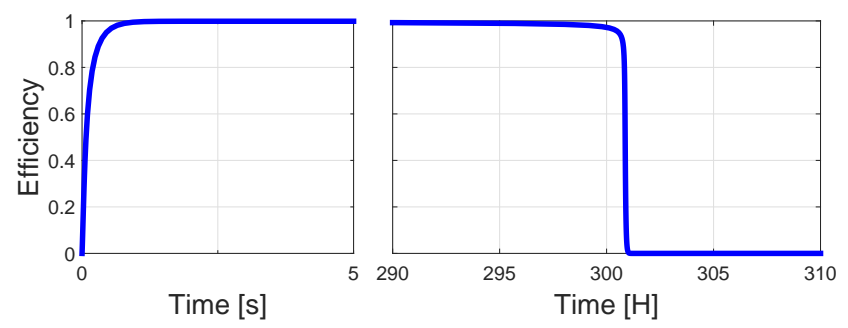

Figure 9: Behavior of efficiency $\eta$ for sharp stage and failure stage. Step input.

Finally, the efficiency $\eta$ of the mechanical power transmission is evaluated in the sharp stage and in the failure stage as depicted in Figure 9. Remark that in the sharp stage the variation of $\eta$ is sharp also. On the other hand, $\eta$ begins to variate before of the total failure and it has a slow variation.

\subsubsection{Variable behavior of input}

A square signal is introduced to represent several cycles of use. In this case $t_{h}$ and $t_{l}$ are the times in which the signal is in high and low respectively. One use cycle is the time in which the contact material deteriorate, i.e. when $\Delta_{v}>0$.

Figure 10 shows the states $v_{1}, v_{2}$ and $D$ with an fixed input of $t_{h}=10000 \mathrm{~s}$ and $t_{l}=10 \mathrm{~s}$ chosen arbitrarily. As a result, $v_{1}$ continues responding to the input signal, but not $v_{2}$. Here is shown that the rolleron-tire system reaches the $D_{\max }$ near to $96.7 \mathrm{~h}$. It means that with multiple cycles of activation, an earlier $D_{\max }$ is reached. Figure 11 shows the behavior of $\alpha$ for the same scenario.

\subsection{Stochastic operational mode}

\subsubsection{Internal mode of randomness}

According to Section 2.4 we can introduce randomness in the model. Firstly, a fixed step input is introduced to the system, which represents a cyclical behavior, where $t_{h}$ and $t_{l}$ are constant.

Figure 12 shows $D$ for 100 simulations. Here $m$ and $b$ are chosen randomly using a normal distribution with mean values of $m_{m}=0.01$ and $b_{m}=10$ respectively and with standard deviations of $\sigma_{m}=0.001$ and $\sigma_{b}=1$ respectively. The different behaviors are 

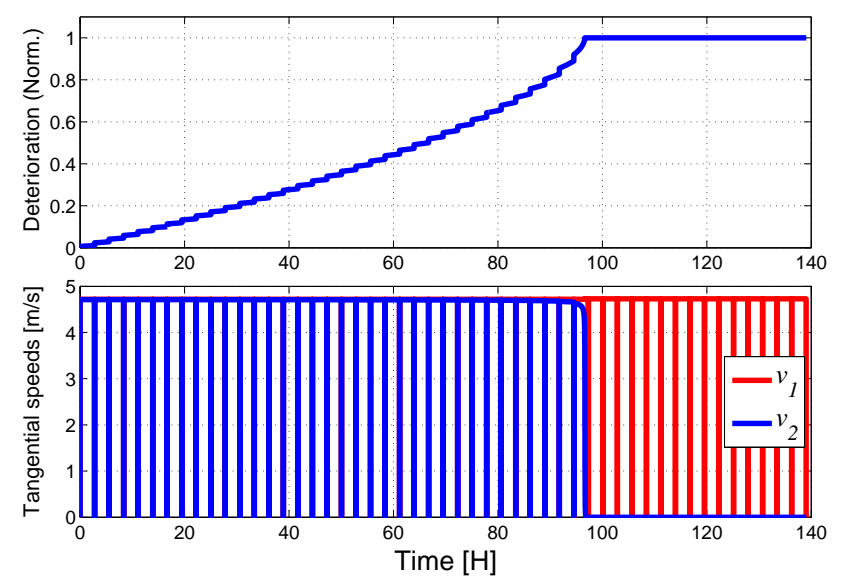

Figure 10: Deterioration $D$, tangential speed of motor $v_{1}$ and tangential speed of wheel $v_{2}$ for a square input with $t_{h}=10000 \mathrm{~s}$ and $t_{l}=10 \mathrm{~s}$. It shows the failure of system at $96.7 \mathrm{~h}$.

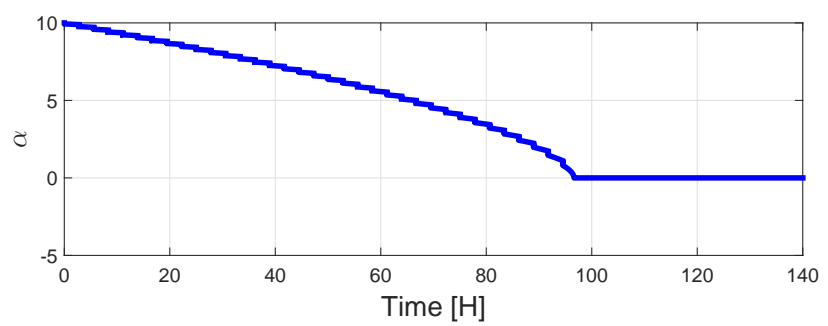

Figure 11: $\alpha$ for or a square input with $t_{h}=10000 \mathrm{~s}$ and $t_{l}=10 \mathrm{~s}$.

compared with an scenario with fixed values of $t_{h}=$ $10000 \mathrm{~s}$ and $t_{l}=10 \mathrm{~s}$.

This simulation allows inferring that if it is possible to know the values of $m$ and $b$ since the beginning then is could be possible have a good estimation of time of failure.

\subsubsection{External mode of randomness}

Figure 13 shows $D$ for 100 simulations. Here $t_{h}$ and $t_{l}$ are chosen randomly using an exponential distribution with mean values of $t_{h}=10000 \mathrm{~s}$ and $t_{l}=10 \mathrm{~s}$ respectively and parameters $b=10$ and $m=0.01$. The different behaviors are compared with an scenario with fixed values of $t_{h}=10000 \mathrm{~s}$ and $t_{l}=10 \mathrm{~s}$.

This kind of external mode of randomness is similar to real operation conditions, for which the $t_{h}$ and

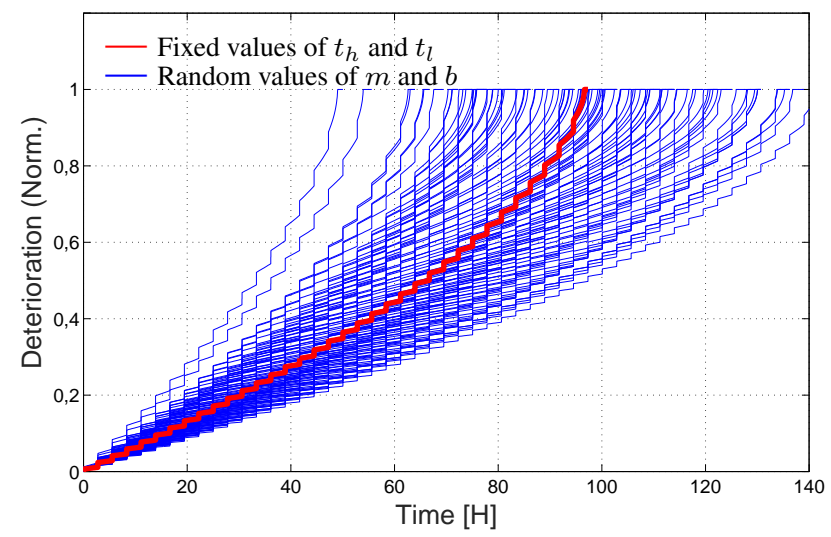

Figure 12: Internal mode of randomness. Blue lines: Deterioration $D$, for 100 random performances. $m$ and $b$ are chosen randomly using a normal distribution. Red line: simulation of performance for fixed values of $t_{h}=10000 \mathrm{~s}$ and $t_{l}=10 \mathrm{~s}$.

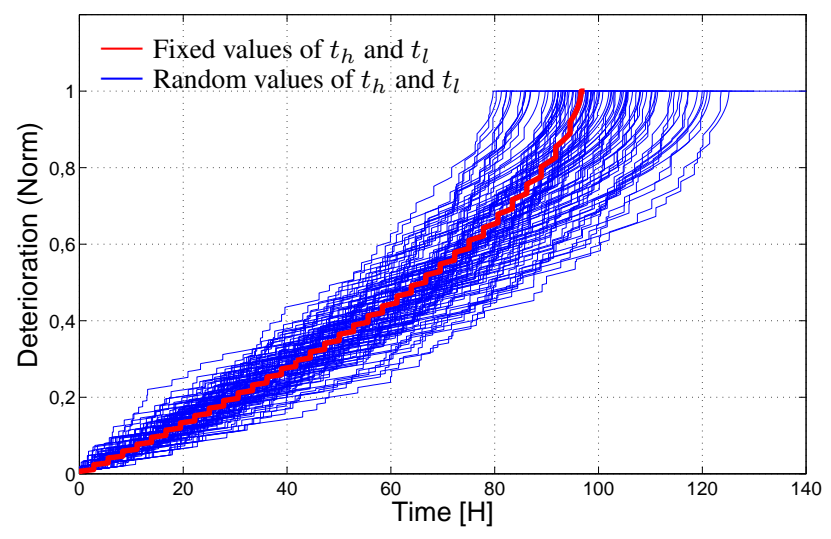

Figure 13: External mode of randomness. Blue lines: Deterioration $D$, for 100 random performances. $t_{h}$ and $t_{l}$ are chosen randomly using an exponential distribution with mean values of $t_{h}=10000 s t_{l}=10 \mathrm{~s}$ respectively. Red line: simulation of performance for fixed values of $t_{h}=10000 \mathrm{~s}$ and $t_{l}=10 \mathrm{~s}$.

$t_{l}$ are unknown. Results shows that despite of the randomness of $t_{h}$ and $t_{l}$ the deterioration behavior follows what seems a normal distribution. It could be useful, for example to estimate the failure time with an acceptable level of uncertainty.

\section{FROM DETERIORATION MODELING TO THE DEVELOPMENT OF A RELIABILITY ADAPTIVE SYSTEM}

Analytical physics-based models of degradation are useful to develop new techniques under the framework of Prognostic Health Management (PHM), which can be defined as the field of activities that links studies of failures in mechanisms to a system life-cycle management. Recently, type 5 of PHM so-called Reliability-Adaptive Systems or RAS has been introduced to its classification for (Rakowsky \& Bertsche 2015).

In some systems, for example in the friction-based ones, the failure is imminent because of deterioration of materials in contact, and the main goal is trying to optimize the use of functioning resources until the failure. In other words, planned actions which tend to initiate changes of behavior of the component, rather than reacting to failures are needed. To achieve that, to lower work load or wear by selecting appropriate optimal system configurations of components is required according to (Meyer \& Sextro 2014). Systems which possess this capability are called Reliability-Adaptive Systems or RAS by (Rakowsky 2006). In other words a reliability control can allow to manage the deterioration of components like in the self-optimizing mechatronic systems, which are, according to (Gausemeier, Rammig, Schäfer, \& Sextro 2014), a class of intelligent technical systems able to autonomously adapt their behavior if user requirements or operating conditions change.

In the framework of PHM modern approaches the proactive actions are focused in system-level and component-level. Then, combination of experienced- 
based, data-driven and model-based approaches (Byington, Watson, Roemer, Galie, McGroarty, \& Savage 2003), seems to be an appropriate way to improve the efficiency of management proactive actions according to (Muller, Suhner, \& Iung 2008). In this context, common goals for the proactive actions are a) the reduction of the probability of unexpected component failures in a reliable way and/or b) the feasible implementation on-line.

In this sense, component-level proactive strategies continue being explored (See for example (Nguyen, Dieulle, \& Grall 2014)). Physics-based models can explain the phenomena of deterioration in a deterministic way, which can be useful to manage the useful lifetime of a component in control loop to get systems type RAS with feasible implementation. This is specially useful in embedded systems due the computational restrictions.

In this paper, we developed a deterioration model linking the system control input and the deterioration behavior, which can be seen as a first step towards the development of a comprehensive reliability adaptive system. The next step is using the information and the features of the model to estimate on-line the deterioration and the model parameters, to synthesize a reliability adaptive control law in order to manage the RAS taking into account jointly the different point of views of reliability, performance and energy efficiency.

\section{CONCLUSIONS}

The proposed model allows analytically to predict an interval of useful lifetime before the total-failure-time of actuator, including stochastic perturbations. The model takes into account the variation of the contact quality coefficient with respect to the deterioration of the contact-surface material, and this deterioration with respect to the difference of the tangential speeds. Results show that the model represents properly and dynamically the deterioration of surfaces depending of the difference of tangential speeds, i.e. the more the difference (sharp motion stage), the more deterioration. In the smooth motion stage, the estimation of deterioration depends on the initial estimation of the contact quality coefficient.

The estimation of contact quality coefficient is supported on the assumptions that the relative speed is known at every time-instant. Results show that if it is possible to know the value of contact quality coefficient at every time-instant, then it could be possible to have a good estimation of the failure time. It could be useful, for example to estimate the failure time with an acceptable level of uncertainty. Additionally, despite of the unknown input conditions, the deterioration behavior follows central tendency. The precision only depends on the size of the uncertainties on the system parameters and future system usages. Therefore, it could be feasible, for example to esti- mate the failure time with an acceptable level of uncertainty.

This model can be probed in close loop scenarios, with the advantage of probing and simulating control techniques on reliability and fault tolerant control areas for a basic mechanical system. This is particularly useful in learning and researching about RAS systems. Indeed, since such a model would link the control and the reliability of the system, it opens the door to develop a control system which allows the device to adapt autonomously its behavior if user requirements or operating conditions change.

\section{REFERENCES}

Armstrong-Helouvry, B. \& C. Canudas de Wit (1996). The Control Handbook, Chapter Friction Modeling and Compensation, pp. 1369. CRS Press and IEEE Press.

Byington, C., M. Watson, M. Roemer, T. Galie, J. McGroarty, \& C. Savage (2003). Prognostic enhancements to gas turbine diagnostic system. Aerospace Conference, 2003. Proceedings. 2003 IEEE 7, 3247 - 3255.

Gausemeier, J., F. J. Rammig, W. Schäfer, \& W. Sextro (2014). Dependability of self-optimizing mechatronic systems. 21954356. Springer.

Geffen, V. (2009). A study of friction models and friction compensation. Technical report, Technische Univ. Eindhoven, The Netherlands.

Huang, S. \& M. Nagurka (2003). Friction modeling of a freespinning bicycle wheel. In ASME (Ed.), STLE/ASME 2003 International Joint Tribology Conference, Number 265 in 2003-TRIB-265, pp. 53-57. American Society of Mechanical Engineers.

Meyer, T. \& W. Sextro (2014). Closed-loop control system for the reliability of intelligent mechatronic systems. In ASME (Ed.), European Conference of the Prognostics and Health Management Society 2014, Number 265 in 2003-TRIB-265, Paderborn, Germany, pp. 53-57. Univ. of Paderborn.

Muller, A., M.-C. Suhner, \& B. Iung (2008). Formalisation of a new prognosis model for supporting proactive maintenance implementation on industrial system. Reliability Engineering \& System Safety 93(2), 234-253.

Nguyen, D., L. Dieulle, \& A. Grall (2014). Remaining useful life estimation of stochastically deteriorating feedback control systems with a random environment and impact of prognostic result on the maintenance process. In Proceedings of the 2nd European Conference of the Prognostics and Health Management Society (PHME14), pp. 1-11. PHM Society.

Popov, V. (2010). Contact Mechanics and Friction: Physical Principles and Applications. Springer Science \& Business Media.

Rakowsky, U. K. (2006). Modelling reliability-adaptive multisystem operation. International Journal of Automation and Computing 3(2), 192-198.

Rakowsky, U. K. \& B. Bertsche (2015). In L. Podofillini, B. Sudret, B. Stojadinovic, E. Zio, and W. Kröger (Eds.), Proc. of ESREL 2015 - Safety and Reliability of Complex Engineered Systems, pp. 2451-2455. Taylor and Francis Group.

Rodriguez, R. (2014). Active force control for electric assist bicycles. Master's thesis, Dep. of Physics and Electrical Engineering, Univ. Grenoble Alpes.

Si, X.-S., W. Wang, C.-H. Hu, \& D.-H. Zhou (2011). Remaining useful life estimation-a review on the statistical data driven approaches. European Journal of Operational Research 213(1), 1-14. 